\title{
Bluetooth-Based Smart Attendance System
}

\author{
Duckki Lee
}

\begin{abstract}
Recently, there have been many attempts to develop offline classrooms to enhance educational efficiency. One of these attempts is the introduction of IT systems in colleges to replace existing attendance management systems. Existing systems authenticate students' lecture attendance by simply using login data, but this has inherent issues involving substitute attendance and not being able to identify whether students are really present in the lecture room. To solve these problems, we have implemented a Smart Attendance System using Bluetooth, which is supported by most smartphones. This system consists of a student's smartphone app, a lecture room terminal (referred to as Raspberry Pi hereafter), and a management web page. This Smart Attendance System is used to manage attendance and related academic administration by using Bluetooth, HTTP, and RESTful (web services that follows the representational state transfer) technologies.
\end{abstract}

Keywords: Android App, Bluetooth, JWT (JSON Web Token), Raspberry Pi, SDP (Service Discovery Protocol), Smart Attendance System

\section{INTRODUCTION}

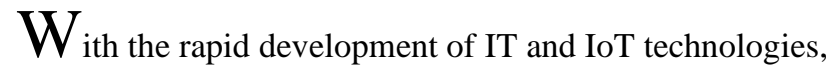
there are many attempts to use these technologies to handle offline administration on campuses. In particular, automatic systems have already been introduced to handle attendance management by many colleges and universities.

In a conventional lecture room, a professor calls every student's name during every lecture and makes a note on an attendance sheet. This requires unnecessary cost with the use of papers and managerial works and even causes inefficiency by taking up lecture time. Further, all attendance sheets need to be processed separately at the end of the semester.

To solve these issues, computer systems have been adopted with a simple website, radio-frequency identification (RFID), biometrics technology, and verification of student ID through terminals. However, such an attendance system does not have guaranteed reliability. In addition, there are implementation problems due to the variety of smartphone models, which may require large implementation costs for special equipment.

This research aims to develop and deploy a Smart Attendance System using Bluetooth, which is supported by almost all smartphones. This Smart Attendance System consists of a smartphone app for checking students'

Revised Manuscript Received on February 05, 2020.

* Correspondence Author

Duckki Lee*, Department of Smart Software, Yonam Institute of Technology, Jinju, Korea. E-mail: dlee@yc.ac.kr

(C) The Authors. Published by Blue Eyes Intelligence Engineering and Sciences Publication (BEIESP). This is an open access article under the CC BY-NC-ND license (http://creativecommons.org/licenses/by-nc-nd/4.0/) attendance and a Bluetooth device (smartphone) for identifying the lecture room, a website for attendance management, and an application programming interface (API) server. It operates as follows. First, an attendance check is requested by Bluetooth communication between the Bluetooth device and a student's smartphone. Then, data are transferred to the API server for processing. With the pairing of the Bluetooth device and the smartphone, attendance status can be checked continuously or periodically. This can contribute to preventing fake attendance and ensure reliable attendance management.

\section{RELATED WORK}

We have investigated existing attendance processing systems using adapted communication technology. The findings are summarized as follows.

The proposed attendance system in [1-6] was implemented with RFID [7-9], which is also called an electronic tag. This was able to identify ID by using RF communication. Utilizing this idea, there were attempts to manage students' attendance using RFID tags attached to student ID cards. However, an ID card can be simply handed to another student, which implies the possibility of substitute attendance. Moreover, it is inconvenient for the student to go to the RFID reader to process attendance. Reliability problems and other issues remain unresolved.

Several authors [10-16] proposed attendance processing systems using near-field communication (NFC) [17-19], which has a bandwidth of $13.56 \mathrm{MHz}$ and is intended for use in short-range wireless communication. Its current data communication speed is $424 \mathrm{kbit} / \mathrm{s}$ and is widely used for traffic cards. The system requires an NFC reader to be attached to a computer installed in the lecture room. After an instructor tags their smartphone to the reader, students are expected to tag theirs for an attendance check.

Although this shows improved cost and efficiency over existing attendance systems, the inconvenience factor remains unresolved, since it requires actions by instructors and students to process attendance. Confusion and complications can be generated by these actions. Fake attendance is a risk factor when students leave in the middle of a lecture.

Some smartphones have compatibility issues with the NFC system; for example, iPhone does not support NFC. To solve these problems, we propose using Bluetooth for our

attendance processing system. Bluetooth [20] is one of the industry standards for private area networks complying with the IEEE 802.15.1 standard. Bluetooth uses the industrial, scientific and medical (ISM) radio band $2.00-2.45 \mathrm{GHz}$. It can communicate wirelessly within a radius of $10-100 \mathrm{~m}$. The transmission speed of Bluetooth is slow, but its power consumption is low.

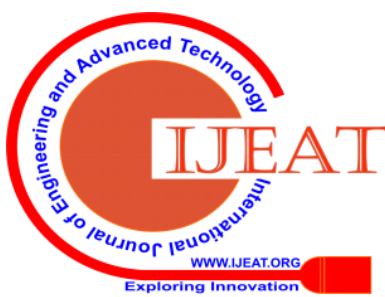




\section{Bluetooth-Based Smart Attendance System}

The system proposed in this research requires pairing within the given radius; it should recognize the radius of the lecture room consistently and thus increase the reliability of students' attendance information. By allowing only one device for each student to apply to the system, substitute attendance can be eliminated.

\section{SMART ATTENDANCE SYSTEM}

Our Smart Attendance System processes students attendance using the Bluetooth communication function in the students' smartphones, which does not require additional equipment. Figure 1 shows the processes of the system.

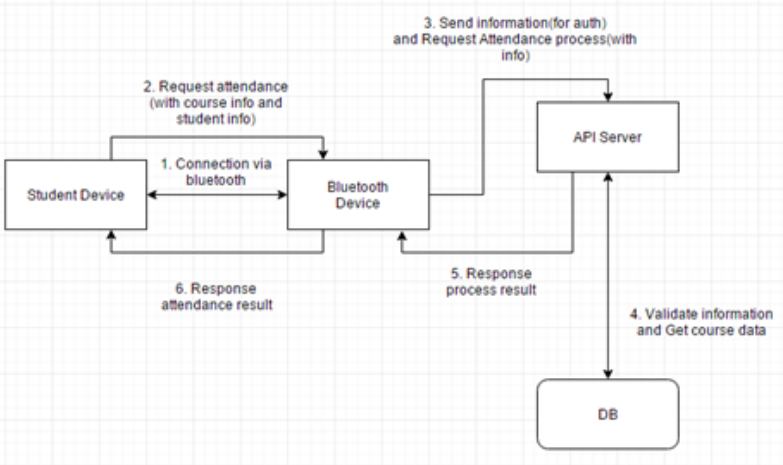

Fig. 1. Process of the Smart Attendance System

The system architecture is presented in Figure 2. It consists of students' smartphones to authenticate students' IDs, a Bluetooth device (Raspberry Pi in our case) identifying the lecture room, an API server with database (DB), and a management website.

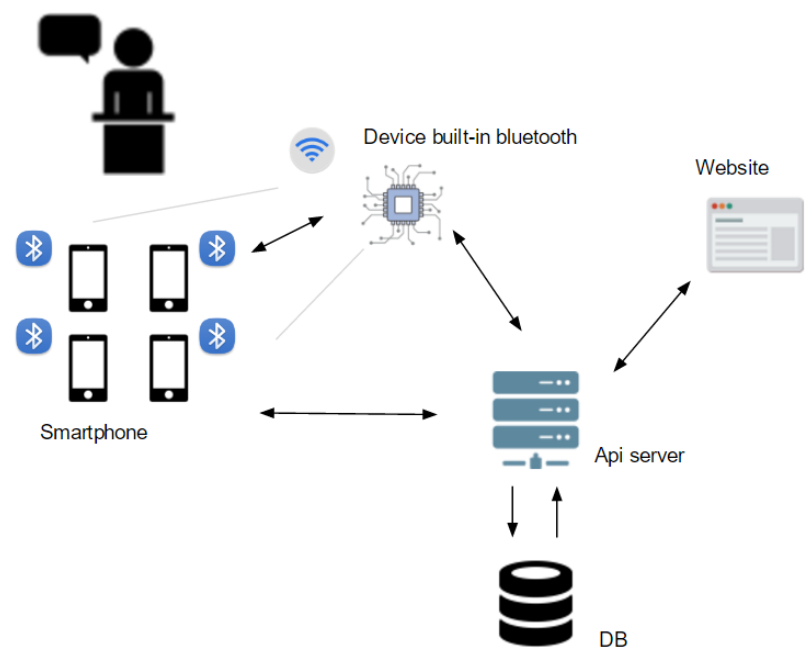

Fig. 2.Architecture of the Smart Attendance System

Students send their information via Bluetooth to the device, which then adds lecture room information and current time when information is received, and sends a processing request to the API server. The server accesses the DB and performs appropriate processing, such as recording attendance and/or lateness based on the information received, and sends the result back to the device. This result is sent to the student's smartphone to provide confirmation of their attendance.

Since the API server operates using HTTP communication, it can be adapted to the system only if Internet and Bluetooth modules are available in all devices. In the API server, an interface is implemented to support three communication links, namely, Bluetooth for attendance confirmation, student devices, and system administrator. If clients only access via HTTP communication, the API server can be used regardless of the hardware and software used. User access rights are classified and managed at student, device, and administrator and/or manager levels. The interfaces supported by the API server are shown in Figure 3.

Student

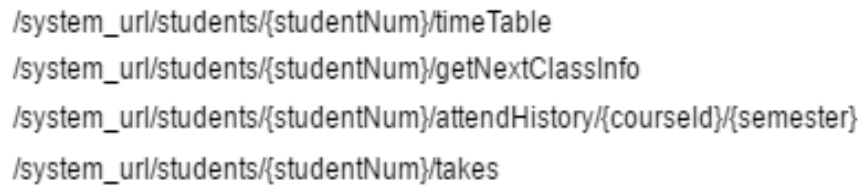

Manager

\section{/system_url/info/getTakesInfo \\ /system_url/info/getAttendStats}

Fig. 3.Interfaces Supported by API Server

The Bluetooth technology [20] in the lecture room device is adapted with service discovery protocol which searches for exposed devices, then performs pairing and communication using RFCOMM protocol.

There are four possible attendance results: attendance, late, absent, and process failure. Absent status is designed to indicate when there is no attendance recorded during lecture hours. The direct connection to the database works only through the API server. Since it uses interfaces supported by the API server, the system is easy to modify to accommodate various models of students' smartphones, or to be migrated according to future system environments or requirements. The system is implemented primarily with Android smartphones, a Bluetooth device (Raspberry Pi is used in this system), and a Spring-based website. For the implementation, MySQL database and Java for both Android and Web were used, while Python was used to develop the program on Raspberry Pi.

Authentication is processed using a representational state transfer (REST) JSON (JavaScript Object Notation) Web Token authentication method [21]. This is an open standard (RFC 7519) that defines a compact and self-contained method for securely transmitting information between parties. The smartphone apps, terminal, and website are all processed with the same authentication method but have different access rights.

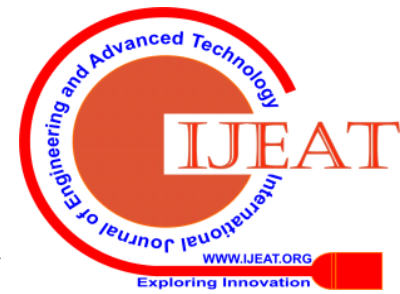


The terminal, Raspberry Pie, was coded in Python and was built using multi-processing and HTTP requests from the pyBluz library [22]. The administration website is implemented using the Spring framework [23]. Figure 4 shows screen shots of the Android application "Here I Am" used by the Smart Attendance System. It indicates (left to right) login, attendance request, timetable, and attendance results.

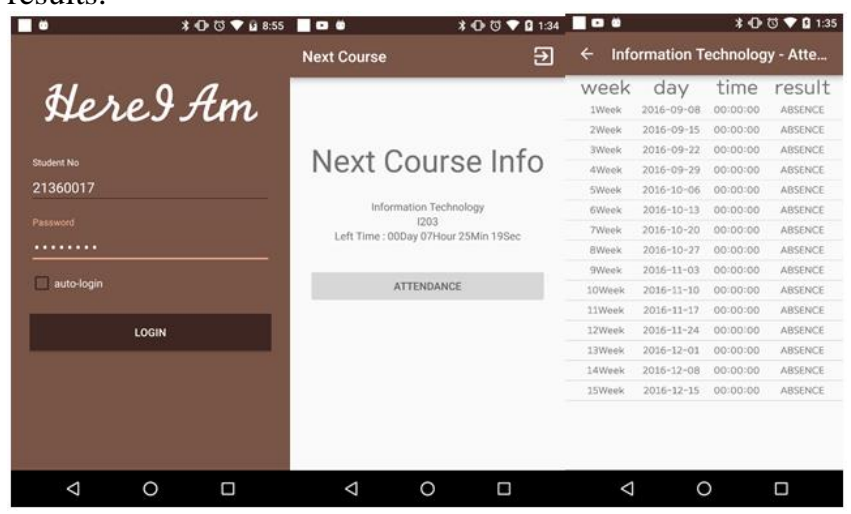

Fig. 4.Screen Shots of "Here I Am"

To prevent fake attendance, we developed a periodic attendance checking method and then conducted an experiment to test if our system can prevent various fraudulent attendance scenarios. We successfully prevent all three major fraudulent scenarios, and details of result are shown in Table-I.

Table -I: Results of Fraudulent Attendance Experiment

\begin{tabular}{|l|l|}
\hline Fake Attendance Scenario & \multicolumn{1}{|c|}{ Result } \\
\hline $\begin{array}{l}\text { Trying to attend outside } \\
\text { the classroom door to } \\
\text { make a fake attendance. }\end{array}$ & $\begin{array}{l}\text { Being outside the } \\
\text { classroom, the attendance } \\
\text { was not accepted. }\end{array}$ \\
\hline $\begin{array}{l}\text { Trying to show attendance } \\
\text { with a friend's ID after } \\
\text { verifying your attendance }\end{array}$ & $\begin{array}{l}\text { If the student logged out in } \\
\text { the middle of a continuous } \\
\text { Bluetooth signal check, } \\
\text { the previous attendance } \\
\text { recording was lost. } \\
\text { Therefore, the attendance } \\
\text { was not accepted. }\end{array}$ \\
\hline $\begin{array}{l}\text { Trying to leave your seat } \\
\text { in the middle of class }\end{array}$ & $\begin{array}{l}\text { Attendance was accepted } \\
\text { when the student was } \\
\text { away for five minutes, but } \\
\text { attendance was not } \\
\text { accepted when he/she was } \\
\text { away for more than ten } \\
\text { minutes. }\end{array}$ \\
\hline
\end{tabular}

\section{CONCLUSION}

There have been many attempts to automate students' attendance management to overcome problems in conventional processing, that is, the individual roll call of students and the processing of the attendance list in the system at later time separately. Thus, IT-based attendance systems based on RFID by using a website and NFC have been attempted. Although these methods successfully reduced manual work, they did not guarantee reliability.

To solve these problems, we have designed and deployed a Smart Attendance System that utilizes the Bluetooth function in students' smartphones. To implement this Smart
Attendance System, a smartphone app and lecture room terminal were developed. Students are expected to use RFCOMM socket communication with Bluetooth-enabled devices in the lecture room. In this new method, students will be able to check their attendance and be able to attend more lecture time. The faculty is able to manage students' attendance in a simpler manner by eliminating manual attendance checks.

Our Smart Attendance System has secured higher reliability than existing systems owing to the specific feature, that is, students should be constantly present within the detection range of Bluetooth and students' reluctance to put their smartphones in the hands of others. Moreover, the equipment can be upgraded immediately with an inexpensive one to enhance compatibility with additional Bluetooth devices, which contributed to minimize costs. The advantage of an API server is that it can easily respond to the requirements of additional capacity to cater for additional

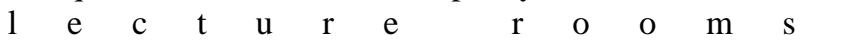
Since the system we propose uses diverse communication technology, it may cause unexpected security issues. Reliability may be ensured by continuous requests to connect to Bluetooth, but it may need additional research in the future on how to deal with unintentional failures of request.

\section{REFERENCES}

1. Arulogun O. T., Olatunbosun, A., Fakolujo O. A., and Olaniyi, O. M "RFID-Based Students Attendance Management System", International Journal of Scientific \& Engineering Research, Volume 4, Issue 2, February 2013.

2. M. Kassim, H. Mazlan, N. Zaini and M. K. Salleh, "Web-based student attendance system using RFID technology," 2012 IEEE Control and System Graduate Research Colloquium, Shah Alam, Selangor, 2012, pp. 213-218.

3. T. Sharma and S. L. Aarthy, "An automatic attendance monitoring system using RFID and IOT using Cloud," 2016 Online International Conference on Green Engineering and Technologies (IC-GET), Coimbatore, 2016, pp. 1-4.

4. M. K. Yeop, M. Z. A. A. Aziz, M. S. R. M. Shah and M. F. A. Kadir, "Smart Attendance System by suing RFID," 2007 Asia-Pacific Conference on Applied Electromagnetics, Melaka, 2007, pp. 1-4.

5. M. Zhi, M. M. Singh, "RFID-Enabled Smart Attendance Managemen System", Future Information Technology, vol. 329, pp. 213-231, 2015

6. T. Toudjeu and P. Z. Sotenga, "Design and implementation of an RFID based smart attendance register," 2017 IEEE AFRICON, Cape Town, 2017, pp. 748-751.

7. Bob Violino "WHAT IS RFID?" in the RFID Journal, 16 Jan, 2005

8. R. Want, "An introduction to RFID technology," in IEEE Pervasive Computing, vol. 5, no. 1, pp. 25-33, Jan.-March 2006.

9. B. Nath, F. Reynolds and R. Want, "RFID Technology and Applications," in IEEE Pervasive Computing, vol. 5, no. 1, pp. 22-24 Jan.-March 2006.

10. Mari Ervasti, Minna Isomursu, and Marianne Kinnula, "Bringing technology into school: NFC-enabled school attendance supervision," in Proceedings of the 8th International Conference on Mobile and Ubiquitous Multimedia. ACM, New York, 2009.

11. T. D. G. F. B. Benyo, B. Sodor, "Student attendance monitoring at the university using nfc," in Wireless Telecommunications Symposium. In press., 2010.

12. M. Bueno-Delgado, P. Pavon-Marino, A. De-Gea-Garcia, and A. DolonGarcia, "The smart university experience: An nfc-based ubiquitous environment," in Innovative Mobile and Internet Services in Ubiquitous Computing (IMIS), 2012 Sixth International Conference on, July 2012, pp. 799-804.

13. B. Benyó, B. Sódor, T. Doktor and G. Fördős, "Student attendance monitoring at the university using NFC," Wireless Telecommunications Symposium 2012, London, 2012, pp. 1-5.

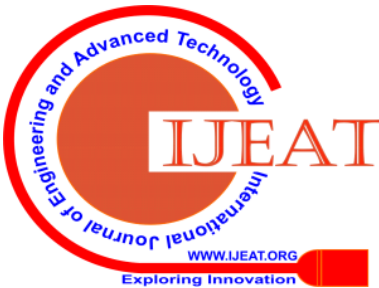




\section{Bluetooth-Based Smart Attendance System}

14. MJL. Fernandez, JG. Fernandez, SR. Aguilar, BS. Selvi, and RG. Crespo, "Control of attendance applied in higher education through mobile NFC technologies," Expert Systems with Applications. vol. 40, pp. 4478-4489, 2013.

15. M. A. Mohandes, "Class Attendance Management System Using NFC Mobile Devices", Intelligent Automation \& Soft Computing, vol. 23, no. 2, pp. 251-259, 2017.

16. M. Baykara, U. Gürtürk and E. Karakaya, "NFC based smart mobile attendance system," 2017 International Conference on Computer Science and Engineering (UBMK), Antalya, 2017, pp. 937-941.

17. R. Want, "Near field communication," in IEEE Pervasive Computing, vol. 10, no. 3, pp. 4-7, July-September 2011.

18. COSKUn, B. OZDENIZCI, K. OK, "A SURVEY ON NEAR FIELD COMMUNICATION (NFC) TECHNOLOGY", WiRELESS PERS. COMMUN., VOL. 71, NO. 3, PP. 2259-2294, 2013.

19. NFC Forum, http://nfc-forum.org/what-is-nfc/about-the-technology/

20. P. Bhagwat, "Bluetooth: technology for short-range wireless apps," in IEEE Internet Computing, vol. 5, no. 3, pp. 96-103, May-June 2001.

21. JWT, http://jwt.io/

22. PyBluez, http://github.com/karulis/pybluez

23. Spring application framework, https://spring.io/

\section{AUTHORS PROFILE}

Duckki Lee is currently an Assistant Professor in the Department of Smart Software, Yonam Institute of Technology. His research interests include mobile computing, smart systems, and Internet of Things. 